\title{
Three dimensional shape and stress monitoring of bulk carriers based on iFEM methodology
}

\author{
Adnan Kefal ${ }^{1,2,3}$, Jimmy Bunga Mayang ${ }^{1}$, Erkan Oterkus ${ }^{1}$, and Mehmet Yildiz ${ }^{2,3,4}$ \\ ${ }^{1}$ Department of Naval Architecture, Ocean and Marine Engineering, University of Strathclyde, \\ 100 Montrose Street Glasgow G4 OLZ, United Kingdom \\ ${ }^{2}$ Integrated Manufacturing Technologies Research and Application Center, Sabanci University, \\ Tuzla, 34956 Istanbul, Turkey \\ ${ }^{3}$ Composite Technologies Center of Excellence, Istanbul Technology Development Zone, \\ Sabanci University-Kordsa Global, Pendik, Istanbul, Turkey \\ ${ }^{4}$ Faculty of Engineering and Natural Sciences, Sabanci University, Tuzla, 34956 Istanbul, \\ Turkey
}

E-mail addresses: adnankefal@sabanciuniv.edu (A. Kefal), bungamayangjimmy@hotmail.com

(J. Bunga Mayang), erkan.oterkus@strath.ac.uk (E. Oterkus), meyildiz@sabanciuniv.edu (M.

Yildiz)

\begin{abstract}
Over the last few years, inverse finite element method (iFEM) is shown to be one of the most robust and general algorithms for the purpose of shape and stress sensing. This study concerns the application of iFEM methodology to a capsize bulk carrier and investigates an appropriate sensor placement configuration for better structural health monitoring of the vessel. The measured uniaxial strain data, e.g. the ones collected from fiber Bragg grating (FBG) sensors, are processed by the developed iFEM framework. For this purpose, hydrodynamic and finite element analyses are performed to generate simulated FBG sensor-strains data for the bulk carrier floating in head sea wave condition. Up to ten percent white noise is added on the numerical strain data to represent experimental strain measurements collected from real FBG sensors. The influence of FBG sensor locations as well as noise level in the strain measurements are examined versus the solution accuracy. Based on the displacement and stress comparison between iFEM and the reference solutions, it was observed that a sparse deployment of FBG sensors is sufficient to predict accurate bending response of the vessel. Hence, practical applicability of iFEM technology together with FBG sensors is demonstrated for the bulk carriers.
\end{abstract}

Keywords: Shape and stress sensing, Structural health monitoring, Inverse Finite Element Method, Fiber Bragg grating sensors, Fiber optic sensing systems, Capsize bulk carrier. 


\section{Introduction}

Marine structures are often exposed to extreme wave loads which can result in structural damages on the structure. In addition, the interaction between sea water and high-strength steel (i.e., the material of most marine structures) can rapidly lead the structure to be corroded. This will then reduce the structural health, integrity, and safety of the structure, while endangering human and environmental safety. Therefore, it is necessary to utilize a structural health monitoring (SHM) system that provides real-time information regarding on-site structural condition.

Various types of monitoring systems have been used on marine vessels, after the International Maritime Organization recommended the use of SHM systems for safety of ships in 1994. For example, class societies including the American Bureau of Shipping (1995, 2015), Det Norske Veritas (1997, 2011), and Lloyds Register (2004) published guidance notes for conventional hull structural monitoring. Moreover, various researchers have investigated several types of SHM systems for marine structures (e.g., Kageyama et al., 1998; Wang et al., 2001; Torkildsen et al., 2005; Murawski et al., 2012; Van der Horst et al., 2013; Phelps and Morris, 2013; Majewska et al., 2014). For more information, see Kefal (2017).

Most of the SHM systems mentioned in the previous paragraph do not take the complexity of boundary conditions and structural topology into account. In addition, none of the existing hull structural monitoring systems recommended by class societies and researchers can be used to monitor three-dimensional (3D) full-field displacements and stresses of a marine vessel. In fact, these current SHM systems only monitor several points on the structure such as two points on the weather deck amidships. The questions which remain unanswered are how much reliable information these SHM systems provide to the master/operator of the ship, in terms of the ships' global structural health, and if any alternative solutions exist. These questions can be answered as follows: (1) as a result of the complex structural topology of marine structures and the dynamic loads (waves and winds), due to the complex statistical features of oceans, global structural health monitoring of a marine structure may be far more challenging than the monitoring offered by the existing hull structural monitoring systems, and (2) the possible solution to this challenge may be achieved by an SHM algorithm that can provide real-time monitoring of displacements and stresses anywhere in 3D space of the structure. 
A key technology of the SHM process, referred to as shape and stress sensing, is real-time computation of a structure's 3D displacement and stress fields using a network of in situ strain sensors and measured strains. Tessler and Spangler (2003, 2005) introduced an innovative technology for shape and stress sensing and called it as "inverse finite element method (iFEM)". The general mathematical concept of the iFEM methodology uses a least-squares variational principle that minimizes the sum of squared errors between the analytical and experimental values of strain measures. Unlike the other inverse methods, the iFEM methodology possesses the following beneficial features that justify the iFEM as a superior shape-sensing algorithm:

- The iFEM methodology does not require any loading and/or material information to reconstruct the $3 \mathrm{D}$ displacement field of the structure.

- The iFEM formulation does not require the entire structure to be installed with strain sensors to monitor the entire structural displacements. Only few locations need to be instrumented with any type of strain sensors such as strain rosettes, strain gauges, fiber optic cables.

- The iFEM methodology is free from complex structural geometry and/or boundary conditions.

- The iFEM algorithm can provide robust, stable, and accurate displacement results even with the strain measurements have inherent errors (e.g., noise).

- The iFEM framework is sufficiently fast for real-time monitoring applications.

Various numerical, theoretical, and experimental research of the iFEM algorithm are available in the literature. To begin with, Stoesz (2013) recently obtained a U.S. patent (US 8,515,675 B2) for shape-sensing system that uses iFEM methodology to monitor a downhole structure. Moreover, Tessler and Spangler (2004) formulated a superior three-node triangular inverse-shell element (iMIN3), Kefal et al. (2016) introduced a robust four-node quadrilateral inverse-shell element (iQS4), and Gherlone et al. (2012) implemented an efficient inverse-frame element. Furthermore, Quach et al. (2005) conducted laboratory experiments using the iMIN3 element. Recently, Tessler et al. (2012) used iMIN3 element for shape-sensing analysis of shell structures undergoing large displacements, while displacement and stress monitoring of various ship types was performed by using the iQS4 element (Kefal and Oterkus, 2016a, 2016b). In addition, Gherlone et al. (2014) examined the capability of their inverse-frame element by performing experimental tests. More recently, the horizon of the iFEM methodology was broaden to shape sensing of laminated composites and sandwich plates (Cerracchio et al., 2015). Kefal et al. (2017) newly enhanced 
Cerracchio's iFEM formulation and developed a novel inverse-shell element, i3-RZT, in order to increase the practical capability of iFEM for composite structures.

To the best of authors' knowledge, the application of iFEM has been so far demonstrated for displacement and stress monitoring of aerospace vehicles (Tessler, 2007; Tessler et al., 2011; Kefal and Oterkus, 2017) and marine structures (Kefal et al., 2015; Kefal and Oterkus, 2015, 2016a, 2016b) only. Yet, none of the marine structure applications included shape and stress sensing of a bulk carrier. This study, therefore, mainly focuses on an application of the iFEM for real-time monitoring of 3D deformations and stresses of a capsize bulk carrier for the first time in the literature. For this purpose, the iQS4/iFEM methodology (Kefal et al., 2016) is employed together with a fiber optic sensing system that uses FBG strain-sensors. In this manner, fine strain measurements can be easily collected in operational conditions of the bulk carrier. To simulate accurate FBG sensor-strain and establish reference solution, the hydrodynamic and FEM analyses are performed for time increments using hydrodynamic panels and high-fidelity discretization of the bulk carrier, respectively. Then, various iFEM case studies are conducted utilizing these simulated FBG strain measurements that involve noise levels up to ten percent. Finally, the comparison between reference solutions and iFEM predictions demonstrated the effect of FBG sensor locations and noise levels on solution accuracy.

\section{The iQS4/iFEM Formulation}

In remainder of this section, the iQS4 element formulation (Kefal et al., 2016), developed on the basis of original iFEM formulation (Tessler and Spangler, 2003, 2005), is briefly summarized. To formulate iQS4 element, Kefal et al. (2016) adopted the kinematic relations of first-order shear deformation theory (FSDT), and interpolated the kinematic variables by using the anisoparametric interpolations (Tessler and Hughes, 1983) and the six displacement degrees-of-freedom (DOF) per node as shown in Fig. 1. Thus, the iQS4 element is robust against both membrane- and bendinglocking occurrence and suitable for shape sensing of complex built-up structures.

Under the assumption of small strains, the in-plane and transverse-shear strains can be defined based on FSDT as 


$$
\left\{\begin{array}{l}
\varepsilon_{11} \\
\varepsilon_{22} \\
\gamma_{12}
\end{array}\right\} \equiv\left\{\begin{array}{l}
e_{1} \\
e_{2} \\
e_{3}
\end{array}\right\}+z\left\{\begin{array}{l}
\kappa_{4} \\
\kappa_{5} \\
\kappa_{6}
\end{array}\right\}
$$

and

$$
\left\{\begin{array}{l}
\gamma_{1 z} \\
\gamma_{2 z}
\end{array}\right\} \equiv\left\{\begin{array}{l}
\gamma_{7} \\
\gamma_{8}
\end{array}\right\}
$$

where the symbol $z \in[-h,+h]$ defines the thickness coordinate of the iQS4 element, the symbols $e_{\alpha}(\alpha=1,2,3), \kappa_{\alpha}(\alpha=4,5,6)$, and $\gamma_{\alpha}(\alpha=7,8)$ denote the membrane strain measures, bending curvatures, and transverse-shear strain measures, respectively. The explicit definitions of these analytic section strains can be written in terms of nodal displacement vector $\left(\mathbf{u}^{e}\right)$ of the iQS4 element as

$\left[\begin{array}{lll}e_{1} & e_{2} & e_{3}\end{array}\right] \equiv\left[\begin{array}{lll}\mathbf{B}_{1} \mathbf{u}^{e} & \mathbf{B}_{2} \mathbf{u}^{e} & \mathbf{B}_{3} \mathbf{u}^{e}\end{array}\right]$,

$\left[\begin{array}{lll}\kappa_{4} & \kappa_{5} & \kappa_{6}\end{array}\right] \equiv\left[\begin{array}{lll}\mathbf{B}_{4} \mathbf{u}^{e} & \mathbf{B}_{5} \mathbf{u}^{e} & \mathbf{B}_{6} \mathbf{u}^{e}\end{array}\right]$,

and

$\left[\begin{array}{ll}\gamma_{7} & \gamma_{8}\end{array}\right] \equiv\left[\begin{array}{ll}\mathbf{B}_{7} \mathbf{u}^{e} & \mathbf{B}_{8} \mathbf{u}^{e}\end{array}\right]$

with

$\mathbf{u}^{e}=\left[\begin{array}{llll}\mathbf{u}_{1}^{e} & \mathbf{u}_{2}^{e} & \mathbf{u}_{3}^{e} & \mathbf{u}_{4}^{e}\end{array}\right]^{T}$

and

$\mathbf{u}_{i}^{e}=\left[\begin{array}{llllll}u_{i} & v_{i} & w_{i} & \theta_{x i} & \theta_{y i} & \theta_{z i}\end{array}\right]^{T}(i=1-4)$

where the matrices $\mathbf{B}_{\alpha}(\alpha=1-8)$ contain derivatives of the shape functions. The explicit form of these matrices can be found in Kefal (2017).

Traditional strain gages and rosettes or advanced fiber optic sensing systems (e.g., FBG strain sensors) can enable us to gather a large amount of highly accurate in situ surface strain measurements (readings). As depicted in Fig. 2, at least two different strain sensors $\left(\boldsymbol{\varepsilon}_{i}^{+}, \boldsymbol{\varepsilon}_{i}^{-}\right)$must be placed along the thickness direction of each particular location, $\mathbf{x}_{i} \equiv(x, y)_{i}(i=1-n)$. For clarity, the exact locations of these sensors and their strain readings can be identified as 
$\boldsymbol{\varepsilon}_{i}^{+}\left(\mathbf{x}_{i}, z_{i}=+h\right)=\left[\begin{array}{lll}\varepsilon_{11}^{+} & \varepsilon_{22}^{+} & \gamma_{12}^{+}\end{array}\right]_{i}$

and

$\boldsymbol{\varepsilon}_{i}^{-}\left(\mathbf{x}_{i}, z_{i}=-h\right)=\left[\begin{array}{lll}\varepsilon_{11}^{-} & \varepsilon_{22}^{-} & \gamma_{12}^{-}\end{array}\right]_{i}$

where the in situ surface strain measures with the superscripts '+' and '-' pertain to the strain rosettes located on the top and bottom surfaces of the plate, respectively. Note that the subscripts (11) and (22) denote the normal strain measurement along the directions $x$ and $y$, whereas the front subscript (12) denotes the shear strain measurement in the $x y$ plane.

Using the strain readings, experimental membrane strain measures and bending curvatures can be computed at the point $\mathbf{x}_{i}$ as (Tessler and Spangler, 2003, 2005)

$$
\left\{\begin{array}{l}
\mathrm{E}_{1} \\
\mathrm{E}_{2} \\
\mathrm{E}_{3}
\end{array}\right\}_{i}=\frac{1}{2}\left\{\begin{array}{l}
\varepsilon_{11}^{+}+\varepsilon_{11}^{-} \\
\varepsilon_{22}^{+}+\varepsilon_{22}^{-} \\
\gamma_{12}^{+}+\gamma_{12}^{-}
\end{array}\right\}_{i}(i=1-n)
$$

and

$$
\left\{\begin{array}{l}
\mathrm{K}_{4} \\
\mathrm{~K}_{5} \\
\mathrm{~K}_{6}
\end{array}\right\}_{i}=\frac{1}{2 h}\left\{\begin{array}{l}
\varepsilon_{11}^{+}-\varepsilon_{11}^{-} \\
\varepsilon_{22}^{+}-\varepsilon_{22}^{-} \\
\gamma_{12}^{+}-\gamma_{12}^{-}
\end{array}\right\}_{i}(i=1-n)
$$

where in situ section strains, $\mathrm{E}_{\alpha}(\alpha=1-3)$ and $\mathrm{K}_{\alpha}(\alpha=4-6)$, correspond to their analytic counterparts, $e_{\alpha}(\alpha=1-3)$ and $\kappa_{\alpha}(\alpha=4-6)$, given by Eqs. (3-4), respectively. In addition, the in situ transverse-shear strain measures that correspond to their analytic counterparts given by Eq. (5), can be symbolically identified by

$$
\mathbf{G}_{i} \equiv\left[\begin{array}{ll}
\Gamma_{7} & \Gamma_{8}
\end{array}\right]_{i}^{T}(i=1-n)
$$

In Eqs. (10-12), the uppercase Greek letters are used to indicate the presence of experimental error (e.g., noise) in the strain measurements. The in situ surface strains cannot be directly used to calculate the $\mathbf{G}_{i}$, however the contributions of $\mathbf{G}_{i}$ are much smaller than that of the bending curvatures in bending theory of thin shells. Therefore, the $\mathbf{G}_{i}$ contributions can be safely omitted from the iFEM formulation for marine structures as they are made of thin shells.

The iFEM methodology establishes a weighted-least-squares functional to match individual analytical section strains with their experimental counterparts as (Tessler and Spangler, 2005) 
$\Phi_{e}\left(\mathbf{u}^{e}\right)=\sum_{\alpha=1}^{8} w_{\alpha} \varphi_{\alpha}\left(\mathbf{u}^{e}\right)$

where the weighting coefficients $w_{\alpha}(\alpha=1-8)$ are positive valued constants corresponding to individual section strains (Kefal et al., 2016). The squared norms given in Eq. (13) can be stated by the Euclidean norms as

$$
\begin{aligned}
& \varphi_{\alpha} \equiv \frac{1}{n} \int_{A} \sum_{i=1}^{n}\left[e_{\alpha}\left(\mathbf{u}^{e}\right)_{i}-\mathrm{E}_{\alpha i}\right]^{2} d A \quad(\alpha=1,2,3) \\
& \varphi_{\alpha} \equiv \frac{(2 h)^{2}}{n} \int_{A} \sum_{i=1}^{n}\left[\kappa_{\alpha}\left(\mathbf{u}^{e}\right)_{i}-\mathrm{K}_{\alpha i}\right]^{2} d A \quad(\alpha=4,5,6) \\
& \varphi_{\alpha} \equiv \frac{1}{n} \int_{A} \sum_{i=1}^{n}\left[\gamma_{\alpha}\left(\mathbf{u}^{e}\right)_{i}-\Gamma_{\alpha i}\right]^{2} d A \quad(\alpha=7,8)
\end{aligned}
$$

On the other hand, if an element has undetermined in situ strain component, the Eqs. (14-16) take on the reduced form as

$$
\begin{aligned}
& \varphi_{\alpha} \equiv \int_{A}\left[e_{\alpha}\left(\mathbf{u}^{e}\right)\right]^{2} d A \quad\left(\mathrm{w}_{\alpha}=\lambda\right),(\alpha=1,2,3) \\
& \varphi_{\alpha} \equiv(2 h)^{2} \int_{A}\left[\kappa_{\alpha}\left(\mathbf{u}^{e}\right)\right]^{2} d A \quad\left(\mathrm{w}_{\alpha}=\lambda\right),(\alpha=4,5,6) \\
& \varphi_{\alpha} \equiv \int_{A}\left[\gamma_{\alpha}\left(\mathbf{u}^{e}\right)\right]^{2} d A \quad\left(\mathrm{w}_{\alpha}=\lambda\right),(\alpha=7,8)
\end{aligned}
$$

for which the corresponding weighting coefficients are adjusted to a small number such as $\lambda=10^{-5}$ or $\lambda=10^{-6}$. Note that integrations in Eqs. (14-19) should be performed over the mid-plane area of an individual iQS4 element, $A$. All strain compatibility relations can be explicitly satisfied by the equation system given above, thus the weighted-least-squares functional can be minimized with respect to $\mathbf{u}^{e}$ giving rise to

$$
\frac{\partial \Phi_{e}\left(\mathbf{u}^{e}\right)}{\partial \mathbf{u}^{e}}=\Gamma^{e} \mathbf{u}^{e}-\boldsymbol{\varepsilon}^{e}=0 \Rightarrow \boldsymbol{\Gamma}^{e} \mathbf{u}^{e}=\boldsymbol{\varepsilon}^{e}
$$

where left-hand-side matrix, $\Gamma^{e}$, doesn't change for a given network of strain sensors and is not dependent on the in situ strain values; right-hand-side vector, $\boldsymbol{\varepsilon}^{e}$, is a function of the in situ strain values; and $\mathbf{u}^{e}$ is the unknown displacement DOF to be solved. As described in Kefal et al. (2016), the element equations (Eq. 20) can be assembled into a global system of equations which can be 
readily solved to obtain the real-time deformed structural shapes of the iFEM model. Finally, constitutive equations lead to the predictions of the 3D and full-field stresses of the structure, providing the key knowledge for real-time damage detection.

\section{Shape and stress sensing of bulk carrier using iFEM methodology}

A capsize bulk carrier that has main particulars given in Table 1 is considered in this study. The mid-ship section used by Yao et al. (2000) is adopted and scaled appropriately (e.g., using characteristic breadth) to fit parallel mid-body of the present bulk carrier model. For clarity, detailed scantlings of the mid-ship section and corrugated transverse bulkhead of the present bulk carrier is provided in Appendix A. Note that, bulk carrier frame of reference is a global Cartesian coordinate system $(\mathrm{X}, \mathrm{Y}, \mathrm{Z})$ whose origin $(0,0,0)$ is aligned vertically with gravity centre of the bulk carrier and located at the design draft. The $\mathrm{X}-$, $\mathrm{Y}-$, and $\mathrm{Z}$-axes are aligned with the forward direction, portside direction, and opposite gravity direction, respectively.

Table 1. General particulars of bulk carrier.

\begin{tabular}{lll}
\hline General particular & Value & Unit \\
\hline Length between perpendiculars & 285 & $\mathrm{~m}$ \\
Breadth & 50 & $\mathrm{~m}$ \\
Depth & 26.9 & $\mathrm{~m}$ \\
Design draft & 19 & $\mathrm{~m}$ \\
Block coefficient & 0.834 & $\mathrm{~m}^{3} / \mathrm{m}^{3}$ \\
Displacement (at design draft) & 231600.2 & tonnes \\
Vertical center of gravity (from baseline) & 16 & $\mathrm{~m}$ \\
Vertical center of buoyancy (from baseline) & 9.84 & $\mathrm{~m}$ \\
Longitudinal center of gravity (from amidships) & 9.43 & $\mathrm{~m}$ \\
Longitudinal center of buoyancy (from amidships) & 9.43 & $\mathrm{~m}$ \\
Radius of gyration around X-axis & 17.385 & $\mathrm{~m}$ \\
Radius of gyration around Y-axis & 70.867 & $\mathrm{~m}$ \\
Radius of gyration around Z-axis & 70.867 & $\mathrm{~m}$ \\
Radius of gyration for roll-yaw product of inertia & 0 & $\mathrm{~m}$ \\
\hline
\end{tabular}


The domain $\mathrm{X} \in[-52.2 \mathrm{~m},+52.2 \mathrm{~m}]$ defines the mid-body of the carrier which is divided into 3 full and 2 half cargo holds using 4 watertight bulkheads. Each hold has length of $26.1 \mathrm{~m}$ while containing uniformly spaced 31 side shell frames, 11 hopper tank frames, 11 double bottom tank frames, and 6 topside tank frames. Thus, the frame spacing for those frames can be calculated as $0.870 \mathrm{~m}, 2.61 \mathrm{~m}, 2.61 \mathrm{~m}$, and $5.22 \mathrm{~m}$, respectively. In addition to the mid-ship section scantlings, the varying thicknesses associated with those frames are also given in Appendix A. Fig. 3 apparently exhibits the structural topology of the parallel mid-body and cargo hold. The material of the structure is high-strength steel with elastic modulus of $210 \mathrm{GPa}$ and Poisson's ratio of 0.3. As depicted in Fig. 4, bulk carrier's total weight is distributed along its length in accordance with the weight representation given by Comstock (1944). Under this loading condition, it is considered that the bulk carrier floats in head sea waves with no forward speed.

The hydrodynamic model is discretized using 6196 flat panels and analyzed utilizing an inhouse panel code (Kefal and Oterkus, 2014). As a result, six DOF motions and oscillatory pressures of the bulk carrier are predicted for wave amplitude of $1 \mathrm{~m}$ and frequencies of 0.1-0.8 rad/s. It has been observed that the oscillatory pressure distribution at the wave frequency of $0.43 \mathrm{rad} / \mathrm{s}$, depicted in Fig. 5, causes the critical vertical bending scenario. Moreover, the heave and pitch motions at $0.43 \mathrm{rad} / \mathrm{s}$ is calculated for time domain of $t \in[0 \mathrm{~s}, 25 \mathrm{~s}]$ as shown in Fig. 6. At any section along the length, the hydrodynamic vertical bending moment, $\mathrm{M}_{\mathrm{Y}}$, can be computed utilizing the heave and pitch motions (Fig. 6), the hydrodynamic pressures (Fig. 5), and the total weight distribution (Fig. 4). The $\mathrm{M}_{\mathrm{Y}}$ moments at three different sections, i.e., $\mathrm{X}_{0}=0 \mathrm{~m}, \mathrm{X}_{1}=-52.2$ $\mathrm{m}$, and $\mathrm{X}_{2}=+52.2 \mathrm{~m}$, are calculated for frequency of $0.43 \mathrm{rad} / \mathrm{s}$ and plotted against time as shown in Fig. 7. The $\mathrm{M}_{\mathrm{Y}}$ moment at the amidships $\left(\mathrm{X}_{0}\right)$ is the greatest load (refer to Fig. 7), thus taken as input load in the following structural analysis.

The in-house FEM code (Kefal and Oterkus, 2014) is employed to predict structural response of the bulk carrier. Mid-body geometry and deformation is symmetric with respect to XZ- and YZplanes, thus only a quarter model is discretized with 204556 shell elements and 1160382 DOF as shown in Fig. 8a. The constraint boundary condition on the symmetry planes can be applied by restraining the normal translations and in-plane rotations. Moreover, the hydrodynamic vertical bending moment can be applied to a node that is truly bonded (multipoint constraint contact) to nodes located at either positions $\mathrm{X}_{1}$ or $\mathrm{X}_{2}$. Note that only a linear static structural analysis is 
conducted for each time increment. As a result, an accurate reference solution is established for each time increment and time history of simulated in situ strain data are obtained.

As opposed to the FEM discretization, a low-fidelity mesh consisted of 22851 iQS4 elements and $57060 \mathrm{DOF}$ is utilized in the following iFEM analysis (refer to Fig. 8b). For each individual iFEM study, two different noise levels, namely 5\% and $10 \%$ noise, are added to the simulated strain data. To calculate the white Gaussian noise corresponding to these noise levels, a built-in function called $\operatorname{awgn()}$ available in the Matlab/Octave toolbox is utilized. In Fig. 9, the top surface strain measurement, $\varepsilon_{11}^{+}$, and in situ bending curvature, $\mathrm{K}_{4}$, at position $(44.8,12.7,7.95)$ with $0 \%$ noise are compared to those with 5\% and 10\% noise. These comparisons show that the added noise levels generate significant differences in the time history of strain measurements.

Once the sensor strains are simulated, the first iFEM analysis of the bulk carrier is performed for one-to-one strain data, i.e., an iQS4 model with top- and bottom-surface strain rosettes per each element of the low-fidelity discretization shown in Fig. 8b. The weighting coefficients $w_{i}(i=7,8)$ are set to a small number $\left(10^{-5}\right)$ as the transverse-shear strain measures are negligibly small due to the thin shell mechanics. On the other hand, all the remaining weighting constants are set as $w_{i}=1(i=1-6)$ because both membrane strain measures and bending curvatures are experimentally determined using the surface strain measurements. In Fig. 10, reference solution and $\mathrm{iFEM}$ predictions for transverse displacement $\left(\mathrm{U}_{\mathrm{Z}}\right)$ and axial stress $\left(\sigma_{\mathrm{X}}\right)$ at position $(52.2,0,-19)$ are plotted against time variation. Note that the iFEM estimates in Fig. 10 are obtained using the strain measurement with $0 \%, 5 \%$, and $10 \%$ of noise, thus predictive capabilities of iFEM/iQS4 methodology is ideally examined for the presence of noisy strain data. These results clearly demonstrates that iFEM can reconstruct sufficiently accurate displacements and stress results in real time even though the strain measurements include a noise level up to $10 \%$.

Although accurate results are obtained in the first iFEM analysis, instrumenting all the iQS4 elements with strain rosettes may be impractical and expensive. Hence, the majority of the strain sensors used in the first case study are eliminated and relatively sparse strain data is utilized to perform the second iFEM analysis of the bulk carrier. To comply with the practical challenge, the strain readings are collected in an experimentally simpler manner by using FBG sensors rather than conventional strain rosettes. In this case study, each green colored iQS4 element depicted in 
Fig. 11 are considered to be installed with FBG strain sensors aligned with axial direction (X-axis) of the bulk carrier. Therefore, only $2.26 \%$ of the total iQS4 elements have been installed with FBG sensors meaning that $97.74 \%$ is strainless inverse elements in the second iFEM analysis. Since there will be only uniaxial strain measurements (along X-axis) available on these green contoured inverse-shell element, a logical arrangement of weighting coefficients is necessary not only for the iQS4 elements with FBG sensors but also for those without FBG. The weighting constants associated with membrane strain measures and bending curvatures are defined as $w_{i}=1(i=1,4)$ and $w_{i}=10^{-5}(i=2,3,5,6)$ for an iQS4 element that is installed with FBG strain sensor. To maintain necessary strain connectivity between the elements that have strain sensors, the weighting coefficients are assigned to $w_{i}=10^{-5}(i=1-8)$ for remaining iQS4 elements which has no in situ strain components. Also, the weighting constants corresponding to the transverse-shear strains are set as $w_{i}=10^{-5}(i=7,8)$ for all iQS4 elements.

Time history of reference and iFEM estimates (i.e., obtained using $0 \%, 5 \%$, and 10\% noisy strain data) for $\mathrm{U}_{\mathrm{Z}}$ displacement and $\sigma_{\mathrm{X}}$ stress at position $(52.2,0,-19)$ are clearly compared in Fig. 12 (a-b), respectively. As can be seen from these plots, the iFEM predictions agree well with the reference solutions, even if using only uniaxial strain measurements (FBG sensor readings) collected from only few locations of the bulk carrier. In fact, this precision is not only valid for a point on the structure but also available for all the points in three-dimension of the structure. To validate this aspect, the contour plots for $U_{Z}$ displacement and $\sigma_{X}$ stress at time $t=8.5 \mathrm{~s}$ are compared between high-fidelity FEM and low-fidelity iFEM analysis as depicted in Figs. 13-14, respectively. In Fig. 13, the FEM and iFEM contours are graphically indistinguishable and the deformed shape of bulk carrier are accurately reconstructed for all noise levels of $0 \%, 5 \%$, and $10 \%$. The percent difference between FEM and iFEM ( $0 \%, 5 \%$, and 10\% noise) predictions for maximum $U_{Z}$ displacement are 1.5\%, 3.5\% and 3.6\%, respectively (Fig. 13), thus showing the superior accuracy of iFEM/iQS4 methodology for shape-sensing analysis.

Besides, similar accuracy is evidenced for the stress-sensing analysis, with percent difference of $2.8 \%$ for the maximum $\sigma_{\mathrm{X}}$ stress between FEM and iFEM ( $0 \%$ noise) analyses (refer to Fig. 14). Nevertheless, iFEM (5\% and 10\%) and FEM analyses produce the maximum $\sigma_{\mathrm{X}}$ stress that 
differs by $14.1 \%$ and $19.1 \%$, respectively. These iFEM predictions remain sufficiently accurate even with the missing strain data in many elements. In Fig. 14, iFEM analysis truly predicts maximum and minimum $\sigma_{\mathrm{X}}$ stress locations for all three noise levels. Hence, these results also confirm the remarkable stress-sensing capabilities of iFEM/iQS4 methodology, especially considering the low-fidelity mesh with sparse and noisy strain measurements.

\section{Conclusions}

Real-time 3D displacements and stresses of a capsize bulk carrier are reconstructed using iFEM/iQS4 methodology together with fiber optic sensing systems. The in situ FBG strain sensor measurements are simulated through a combined hydrodynamic and FEM analysis. Using the simulated sensor-strains, various low-fidelity iFEM analyses of the parallel mid-body are performed to identify effective locations of FBG sensor network. In addition, practical utility of the iFEM technology is explored by including 5\% and 10\% noise levels (inherent errors) in the measured strain data. It has been demonstrated that iFEM methodology together with FBG sensor measurements can enable to monitor accurate transverse displacements and axial stresses of the bulk carriers in real time, even if a relatively sparse strain data subjected to experimental noise is used. Hence, the numerical results confirmed that iFEM is a practical shape- and stress-sensing technology to use in structural health monitoring of bulk carriers. Finally, this study overall can be very useful to configure a practical/viable network of FBG sensors to use in iFEM analyses of bulk carriers. 


\section{Appendix A}

Detailed structural dimensions of the bulk carrier's mid-ship section, web section, and corrugated bulkhead are presented in Figs. A1-A3, respectively. Moreover, the structural scantlings of the stiffeners are listed in Table A1.

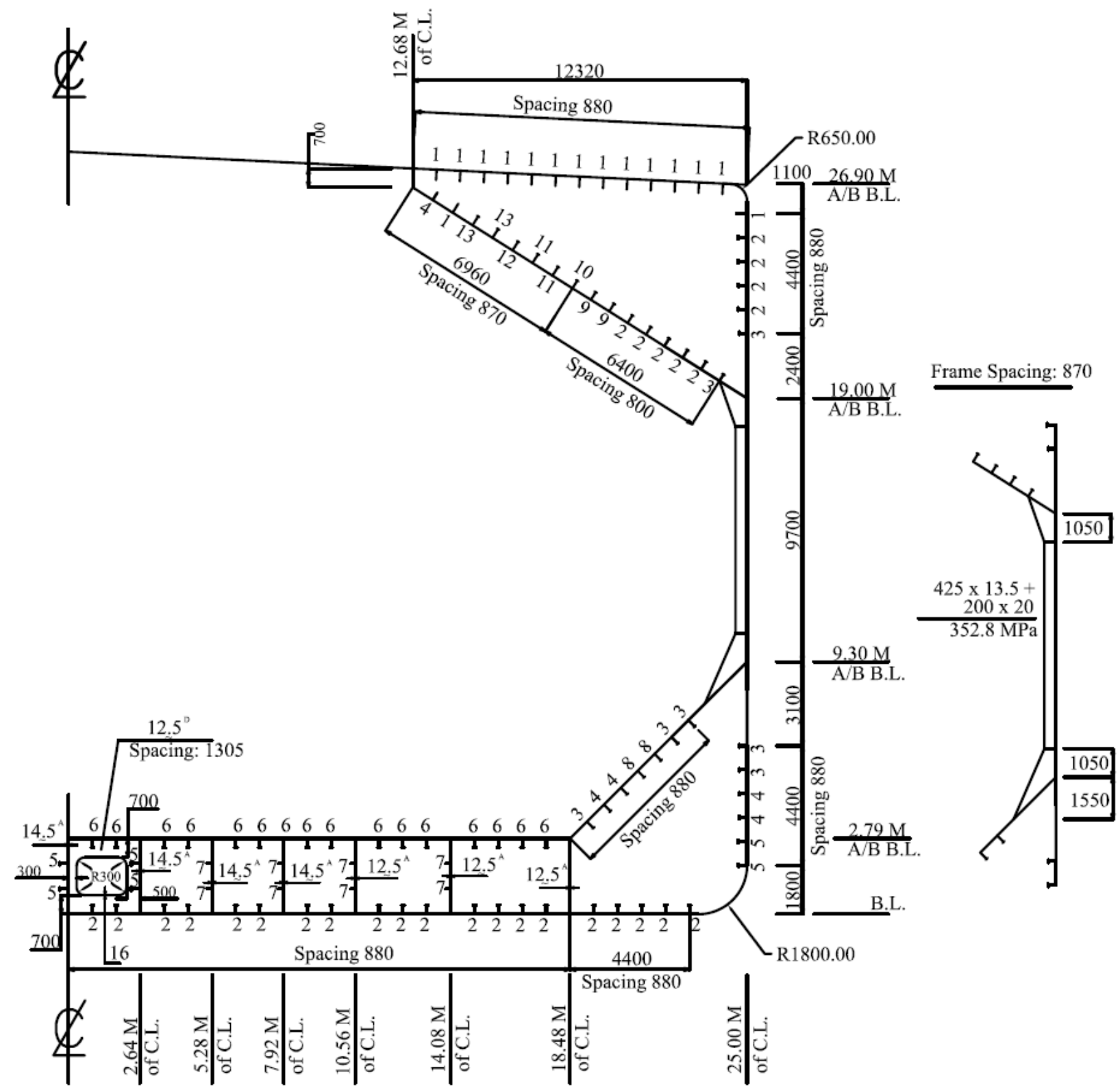

Fig. A1. Structural dimensions [mm] of bulk carrier's mid-ship section 


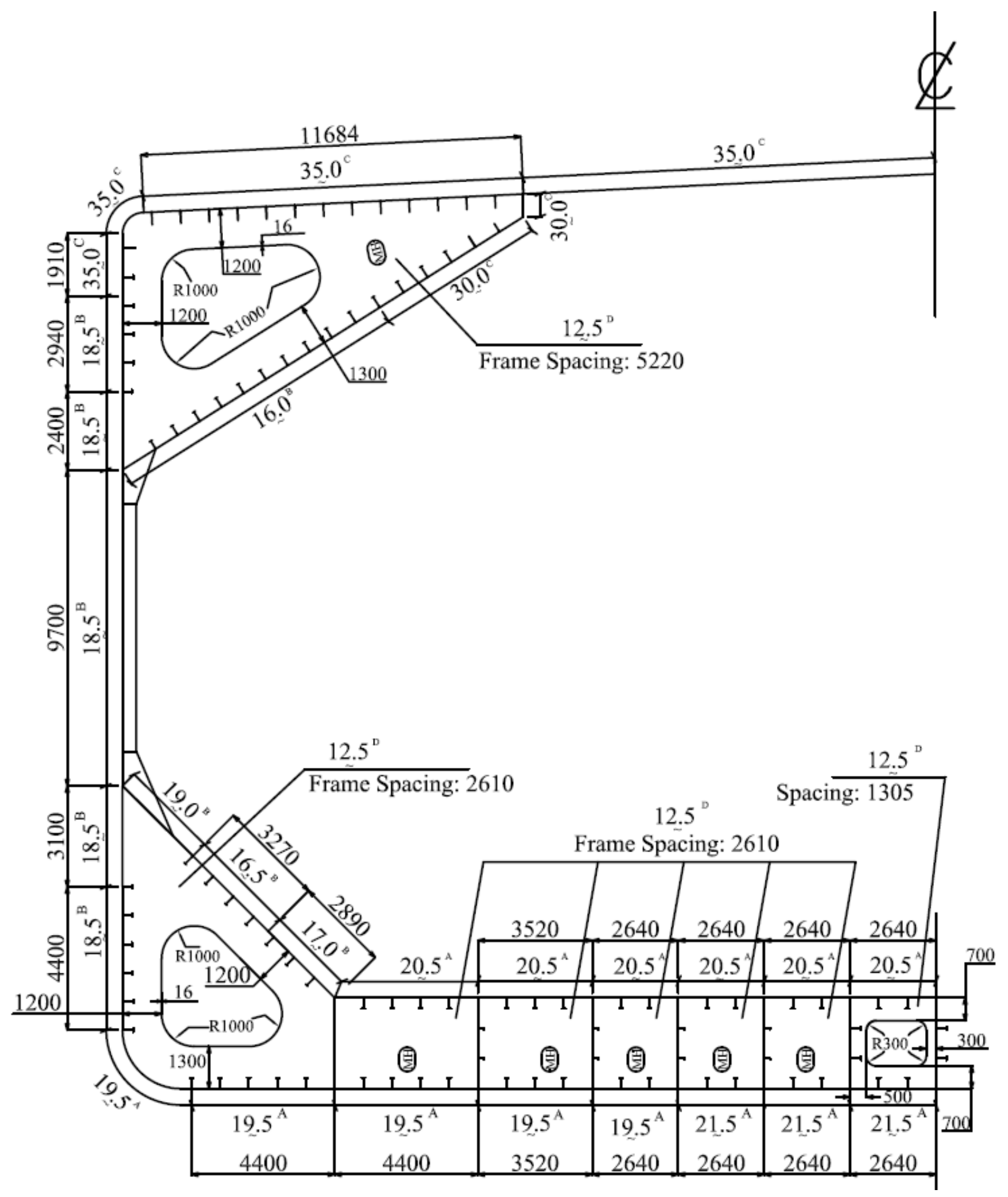

Fig. A2. Structural dimensions [mm] of bulk carrier's web section 


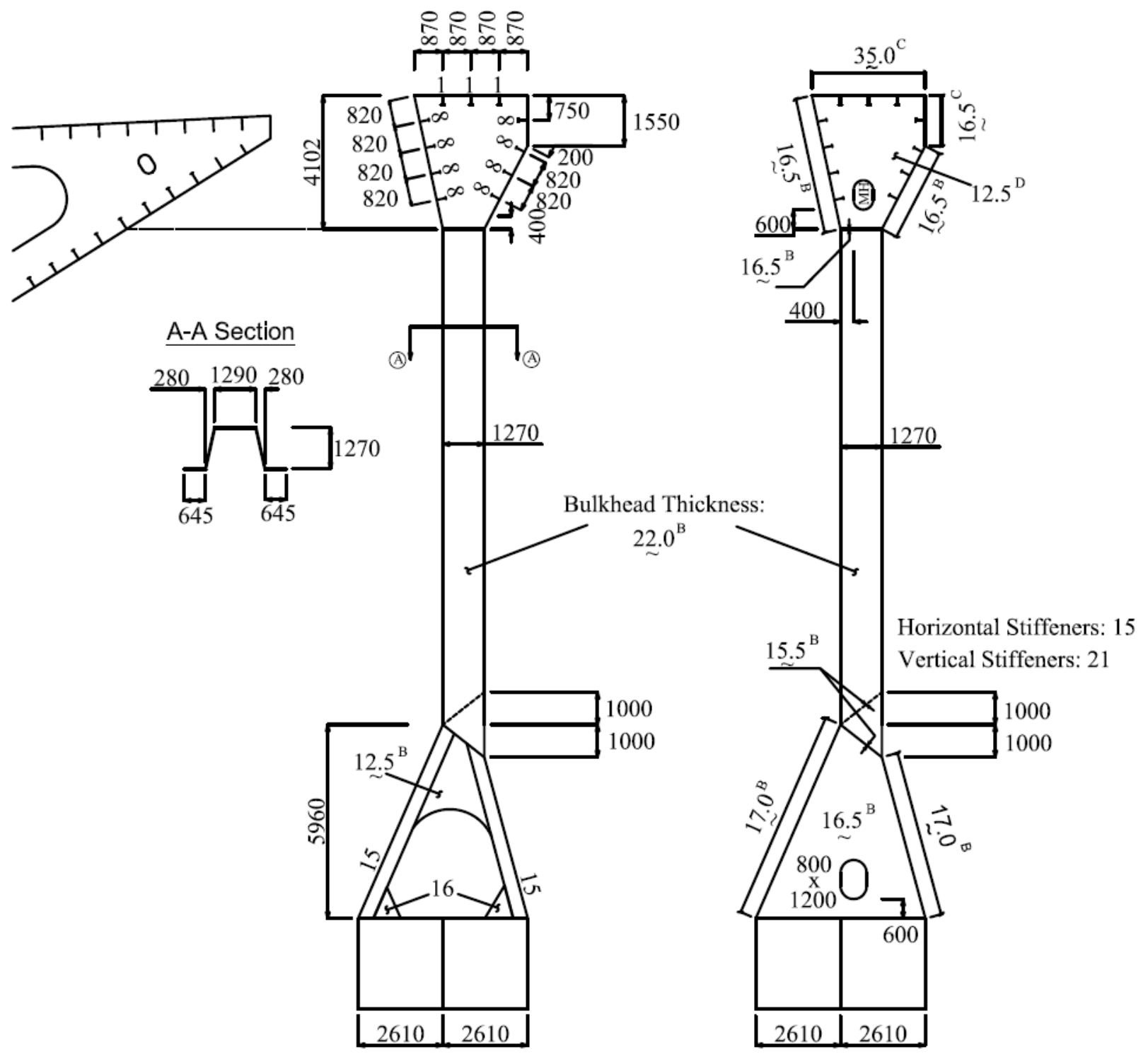

Fig. A3. Structural dimensions [mm] of bulk carrier's corrugated bulkhead 
Table A1. Structural scantlings of stiffeners depicted in Figs. A1-A3.

\begin{tabular}{|c|c|c|c|}
\hline Stiff No. & Properties (in mm) & Type & $\begin{array}{c}\text { Yield } \\
\text { Strength (MPa) }\end{array}$ \\
\hline 1 & $390 \times 27$ & Flat Bar & 392.0 \\
\hline 2 & $333 \times 9+100 \times 16$ & Tee Bar & 352.8 \\
\hline 3 & $283 \times 9+100 \times 14$ & Tee Bar & 352.8 \\
\hline 4 & $283 \times 9+100 \times 18$ & Tee Bar & 352.8 \\
\hline 5 & $333 \times 9+100 \times 17$ & Tee Bar & 352.8 \\
\hline 6 & $283 \times 9+100 \times 16$ & Tee Bar & 352.8 \\
\hline 7 & $180 \times 32.5 \times 9.5$ & Bulb Bar & 235.2 \\
\hline 8 & $283 \times 9+100 \times 17$ & Tee Bar & 352.8 \\
\hline 9 & $333 \times 9+100 \times 18$ & Tee Bar & 352.8 \\
\hline 10 & $333 \times 9+100 \times 19$ & Tee Bar & 352.8 \\
\hline 11 & $383 \times 10+100 \times 18$ & Tee Bar & 352.8 \\
\hline 12 & $383 \times 10+100 \times 21$ & Tee Bar & 352.8 \\
\hline 13 & $390 \times 27$ & Tee Bar & 392.0 \\
\hline 14 & $125 \times 12$ & Flat Bar & 235.2 \\
\hline 15 & $430 \times 10$ & Flat Bar & 352.8 \\
\hline 16 & $850 \times 15$ & Flat Bar & 352.8 \\
\hline
\end{tabular}




\section{References}

American Bureau of Shipping (1995). Guide for Hull Condition Monitoring Systems.

American Bureau of Shipping (2015). Guide for Hull Condition Monitoring Systems.

Cerracchio, P., Gherlone, M., Di Sciuva, M., and Tessler, A. (2015). A novel approach for displacement and stress monitoring of sandwich structures based on the inverse finite element method. Composite Structures, 127: 69-76.

Comstock, J.P. (1944). Introduction to Naval Architecture. Simmons-Boardman Publishing Corporation, New York.

Det Norske Veritas (1997). Hull monitoring system. DNV Rules for Classification of Ships, Part 6, Chapter 11.

Det Norske Veritas (2011). Hull monitoring systems. DNV Rules for Classification of Ships/High Speed, Light Craft and Naval Surface Craft, Part 6, Chapter 11.

Gherlone, M., Cerracchio, P., Mattone, M., Di Sciuva, M., and Tessler, A. (2012). Shape sensing of 3D frame structures using an inverse finite element method. International Journal of Solids and Structures, 49(22): 3100-3112.

Gherlone, M., Cerracchio, P., Mattone, M., Di Sciuva, M., and Tessler, A. (2014). An inverse finite element method for beam shape sensing: theoretical framework and experimental validation. Smart Materials and Structures, 23(4), 045027.

Kageyama, K., Kimpara, I., Suzuki, T., Ohsawa, I., Murayama, H., and Ito, K. (1998). Smart marine structures: an approach to the monitoring of ship structures with fiber-optic sensors. Smart Materials and Structures, 7(4): 472-478.

Kefal, A., and Oterkus, E. (2014). D3.3 (WP3) - Hydrodynamic and structural analysis. Public Deliverable, The INCASS Project (FP7/2007-2013 grant agreement no 605200).

Kefal, A., and Oterkus, E. (2015). Structural health monitoring of marine structures by using inverse finite element method. In C. Guedes Soares and R.A. Shenoi (Eds.), Analysis and Design of Marine Structures V, pp. 341-349, Taylor and Francis Group, London.

Kefal, A., Hizir, O., and Oterkus, E. (2015). A smart system to determine sensor locations for 
structural health monitoring of ship structures. Proceedings of 9th International Workshop on Ship and Marine Hydrodynamics. Glasgow, Scotland.

Kefal, A., and Oterkus, E. (2016a). Displacement and stress monitoring of a chemical tanker based on inverse finite element method. Ocean Engineering, 112: 33-46.

Kefal, A., and Oterkus, E. (2016b). Displacement and stress monitoring of a Panamax containership using inverse finite element method. Ocean Engineering, 119: 16-29.

Kefal, A., Oterkus, E., Tessler, A., and Spangler, J.L. (2016). A quadrilateral inverse-shell element with drilling degrees of freedom for shape sensing and structural health monitoring. Engineering Science and Technology, an International Journal, 19: 1299-1313.

Kefal, A., and Oterkus, E. (2017). Shape sensing of aerospace structures by coupling of isogeometric analysis and inverse finite element method. Proceedings of the 58th AIAA/ASCE/AHS/ASC Structures, Structural Dynamics, and Materials Conference. Grapevine, TX.

Kefal, A. (2017). Structural Health Monitoring of Marine Structures by using Inverse Finite Element Method (Doctoral dissertation). The University of Strathclyde.

Kefal, A., Tessler, A., and Oterkus, E. (2017). An enhanced inverse finite element method for displacement and stress monitoring of multilayered composite and sandwich structures. Under review in Composite Structures.

Majewska, K., Mieloszyk, M., Ostachowicz, W., and Król, A. (2014). Experimental method of strain/stress measurements on tall sailing ships using Fibre Bragg Grating sensors. Applied Ocean Research, 47: 270-283.

Murawski, L., Ostachowicz, W., Opoka, S., Mieloszyk, M., and Majewska, K. (2012). Practical application of monitoring system based on optical sensors for marine constructions. Key Engineering Materials, 518: 261-270.

Phelps, B., and Morris, B. (2013). Review of hull structural monitoring systems for navy ships. DSTO-TR-2818.

Quach, C.C., Vazquez, S.L., Tessler, A., Moore, J.P., Cooper, E.G., and Spangler, J.L. (2005). Structural anomaly detection using fiber optic sensors and inverse finite element method. 
Proceedings of AIAA Guidance, Navigation, and Control Conference and Exhibit. San Francisco, California.

Stoesz, C.W. (2013). Method for Analyzing Strain Data. US 8,515,675 B2.

Tessler, A., and Hughes, T.J.R. (1983). An improved treatment of transverse shear in the Mindlintype four-node quadrilateral element. Computer Methods in Applied Mechanics and Engineering, 39(3): 311-335.

Tessler, A., and Spangler, J.L. (2003). A variational principal for reconstruction of elastic deformation of shear deformable plates and shells. NASA TM-2003-212445.

Tessler, A., and Spangler, J.L. (2004). Inverse FEM for full-field reconstruction of elastic deformations in shear deformable plates and shells. Proceedings of 2nd European Workshop on Structural Health Monitoring. Munich, Germany.

Tessler, A., and Spangler, J.L. (2005). A least-squares variational method for full-field reconstruction of elastic deformations in shear-deformable plates and shells. Computer Methods in Applied Mechanics and Engineering, 194(2): 327-339.

Tessler, A. (2007). Structural analysis methods for structural health management of future aerospace vehicles. Key Engineering Materials, 347: 57-66.

Tessler, A., Spangler, J.L., Gherlone, M., Mattone, M., and Di Sciuva, M. (2011). Real-time characterization of aerospace structures using onboard strain measurement technologies and inverse finite element method. Proceedings of the 8th International Workshop on Structural Health Monitoring. Stanford, CA.

Tessler, A., Spangler, J.L., Gherlone M., Mattone M., and Di Sciuva, M. (2012). Deformed shape and stress reconstruction in plate and shell structures undergoing large displacements: application of inverse finite element method using fiber bragg grating strains. 10th World Congress on Computational Mechanics, Sao Paulo, Brazil. Retrieved from https://ifem.larc.nasa.gov/publi cations/2012-WCCM-Brazil-Tessler.pdf.

Torkildsen, H.E., Grovlen, A., Skaugen, A., Wang, G., Jensen, A.E., Pran, K., and Sagvolden, G. (2005). Development and applications of full-scale ship hull health monitoring systems for the Royal Norwegian Navy. Proceedings of Recent Developments in Non-Intrusive Measurement Technology for Military Application on Model- and Full-Scale Vehicles, pp. 
1-14. Neuilly-sur-Seine, France.

Van der Horst, M.P., Kaminski, M.L., and Puik, E. (2013). Methods for sensing and monitoring fatigue cracks and their applicability for marine structures. Proceedings of the 23rd International Offshore and Polar Engineering Conference. International Society of Offshore and Polar Engineers. Anchorage, Alaska.

Vazquez, S.L., Tessler, A., Quach, C.C., Cooper, E.G., Parks, J., and Spangler J.L. (2005). Structural health monitoring using high-density fiber optic strain sensor and inverse finite element methods. NASA TM-2005-213761.

Wang, G., Pran, K., Sagvolden, G., Havsgard, G.B., Jensen, A.E., Johnson, G.A., and Vohra, S.T. (2001). Ship hull structure monitoring using fibre optic sensors. Smart Materials and Structures, 10(3): 472-478.

Yao, T., Astrup, O.C., Caridis, P., Chen, Y.N., Cho, S.R., Dow, R.S., Niho, O., and Rigo, P. (2000). Report of committee VI. 2, ultimate hull girder strength. Proceedings of the 14th International Ship and Offshore Structures Congress, pp. 321-391. Nagasaki, Japan. 


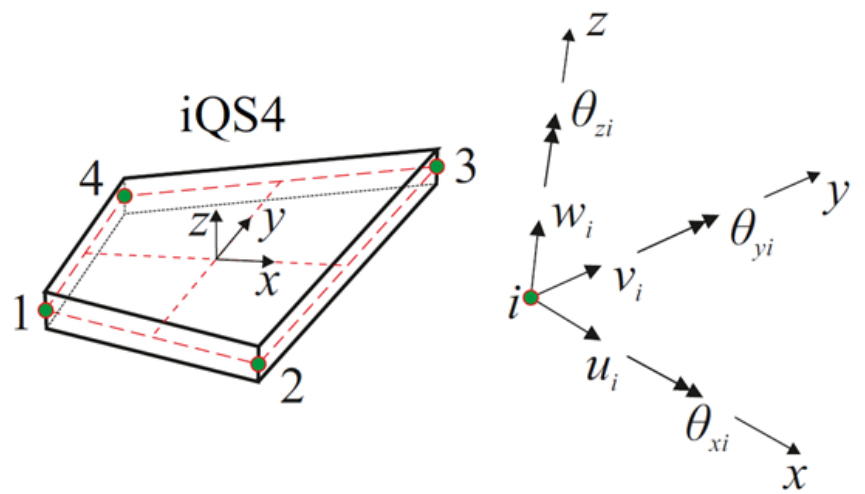

Fig. 1. The iQS4 element and nodal DOF corresponding to local coordinates.

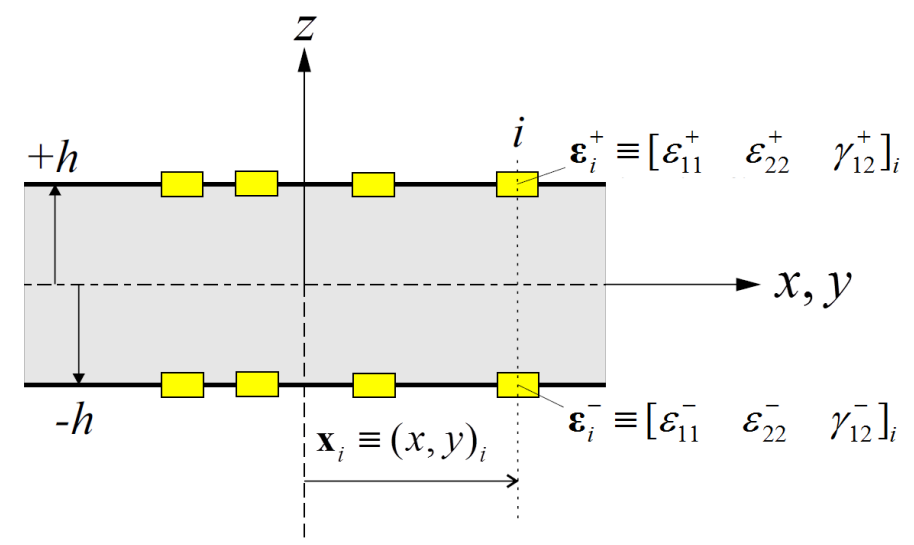

Fig. 2. Surface strains measured by strain rosettes at discrete locations $\left(\mathbf{x}_{i}, \pm h\right)$.

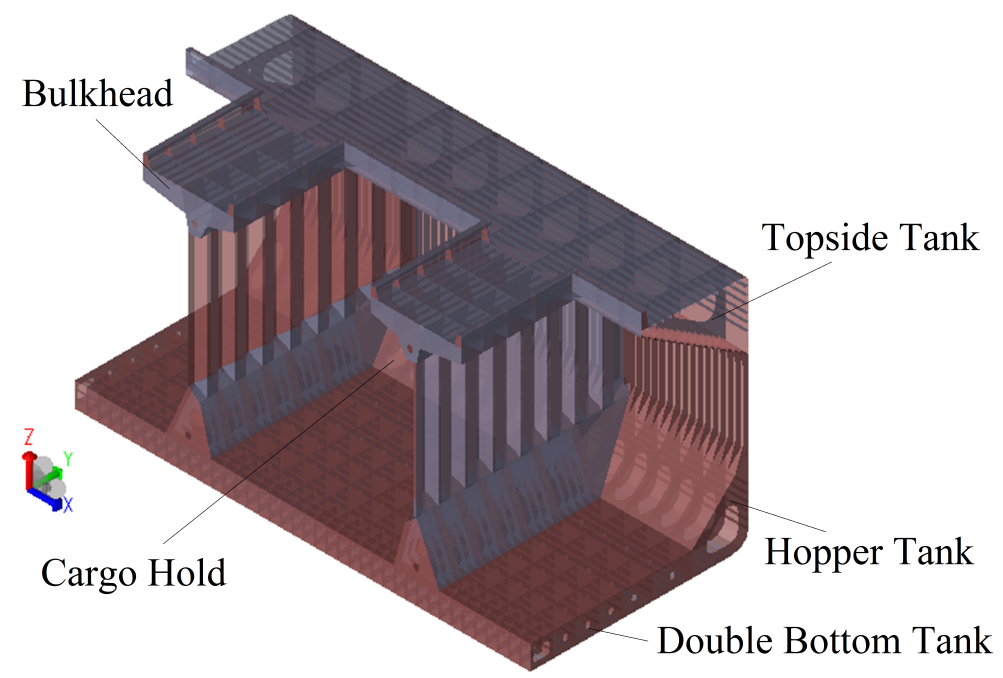

Fig. 3. Bulk carrier cargo model. 


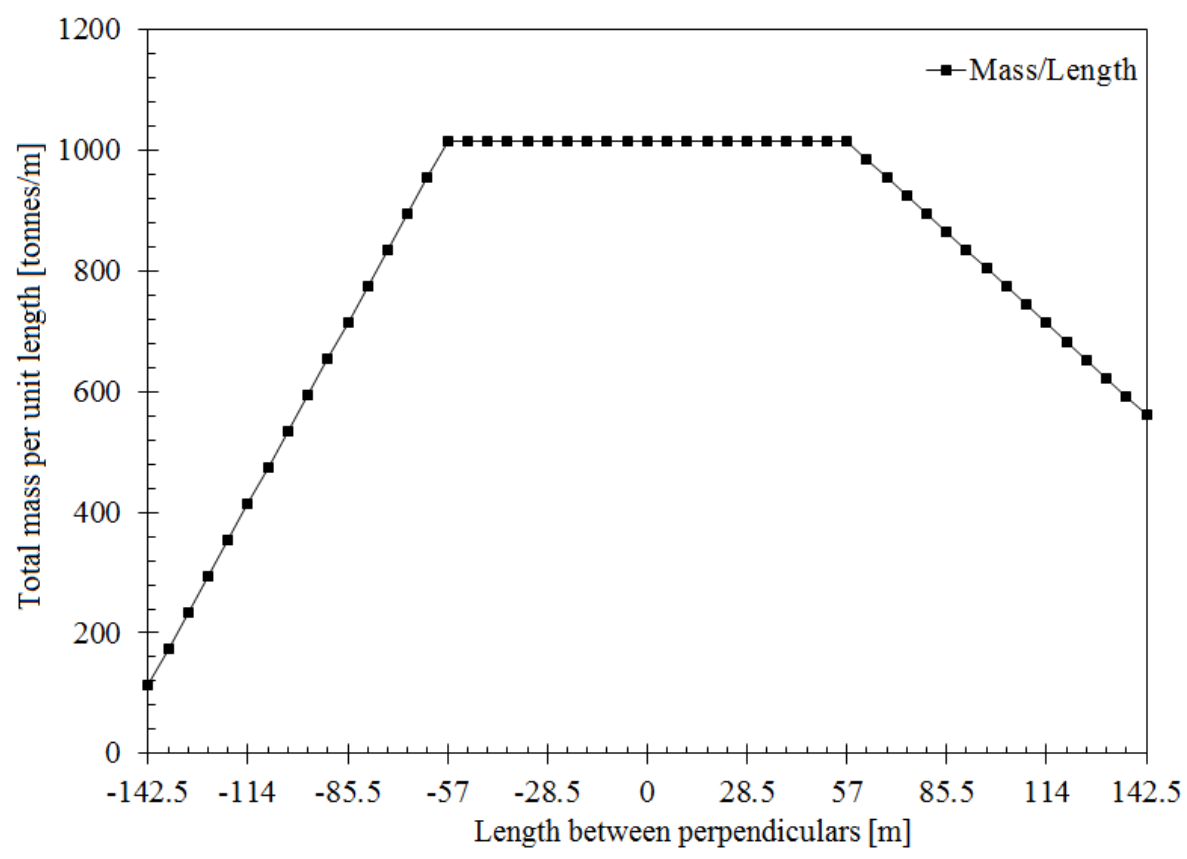

Fig. 4. Total mass distribution of the bulk carrier.

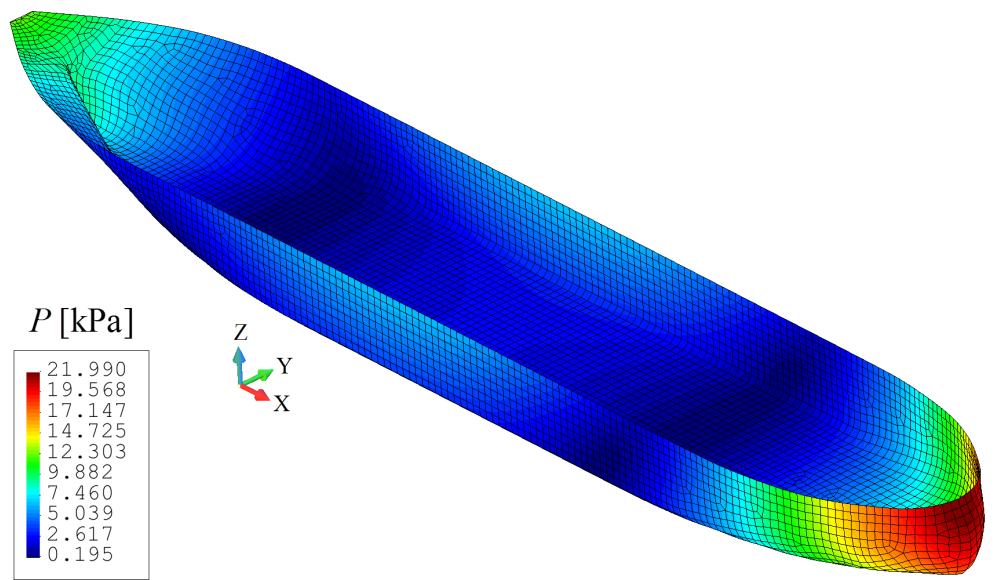

Fig. 5. Contour plot of total hydrodynamic pressure, $P$, acting on the bulk carrier for wave frequency of $0.43 \mathrm{rad} / \mathrm{s}$. 


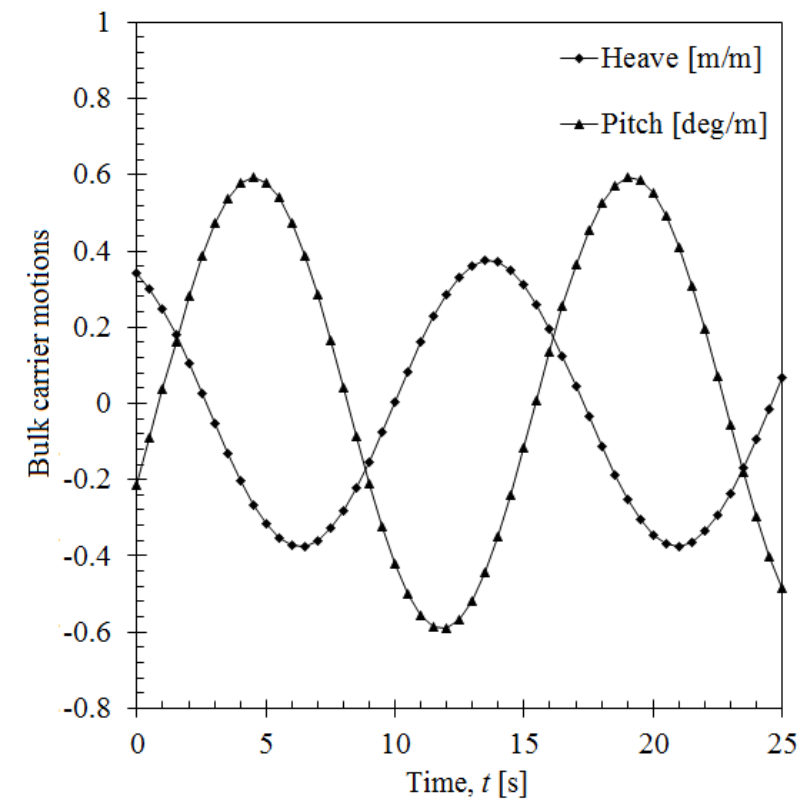

Fig. 6. Time history of the bulk carrier's heave and pitch motions corresponding to wave frequency of $0.43 \mathrm{rad} / \mathrm{s}$.

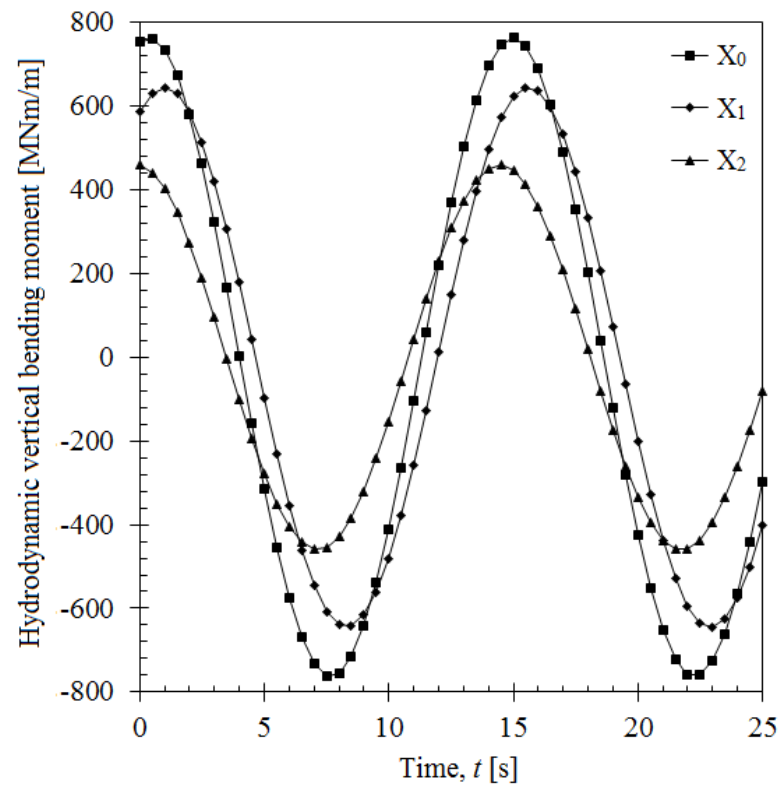

Fig. 7. Time history of hydrodynamic vertical bending moments at three different sections, i.e., $X_{0}=0 \mathrm{~m}, \mathrm{X}_{1}=-52.2 \mathrm{~m}$, and $\mathrm{X}_{2}=+52.2 \mathrm{~m}$, of the bulk carrier. 


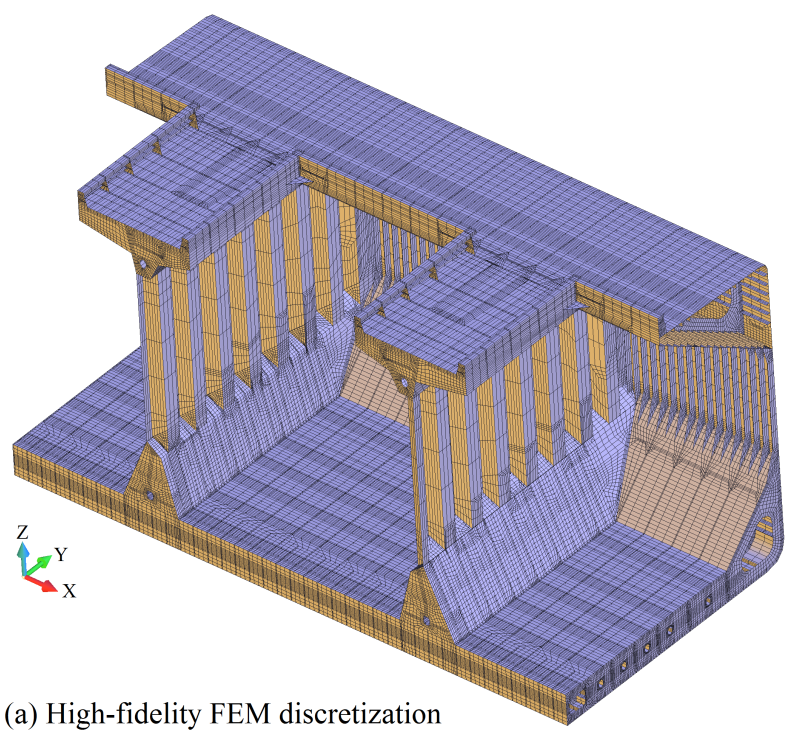

(a) High-fidelity FEM discretization

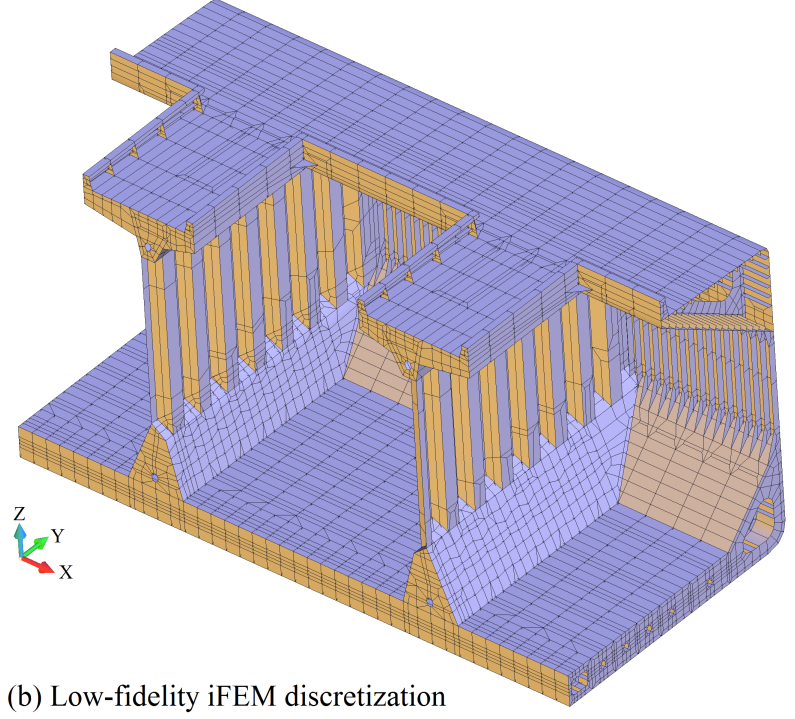

Fig. 8. Discretization of one-fourth of the parallel mid-body: (a) High-fidelity FEM discretization; (b) Low-fidelity iFEM discretization. 

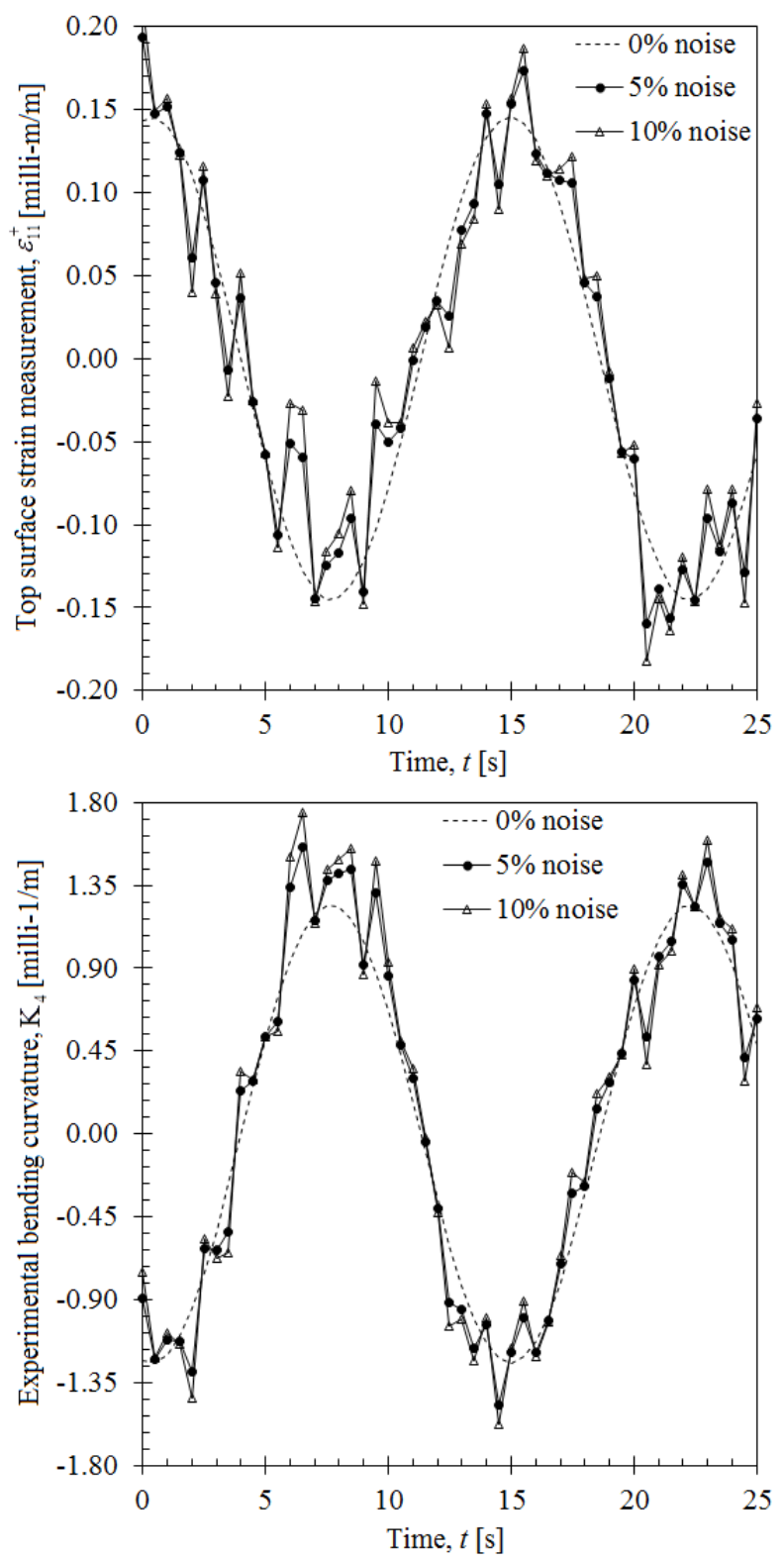

(a)

(b)

Fig. 9. Time history of strain measurements at position $(44.8,12.7,7.95)$ with $0 \%, 5 \%$, and $10 \%$ noise: (a) Top surface strain measurement, $\varepsilon_{11}^{+}$; (b) Experimental bending curvature, $\mathrm{K}_{4}$. 

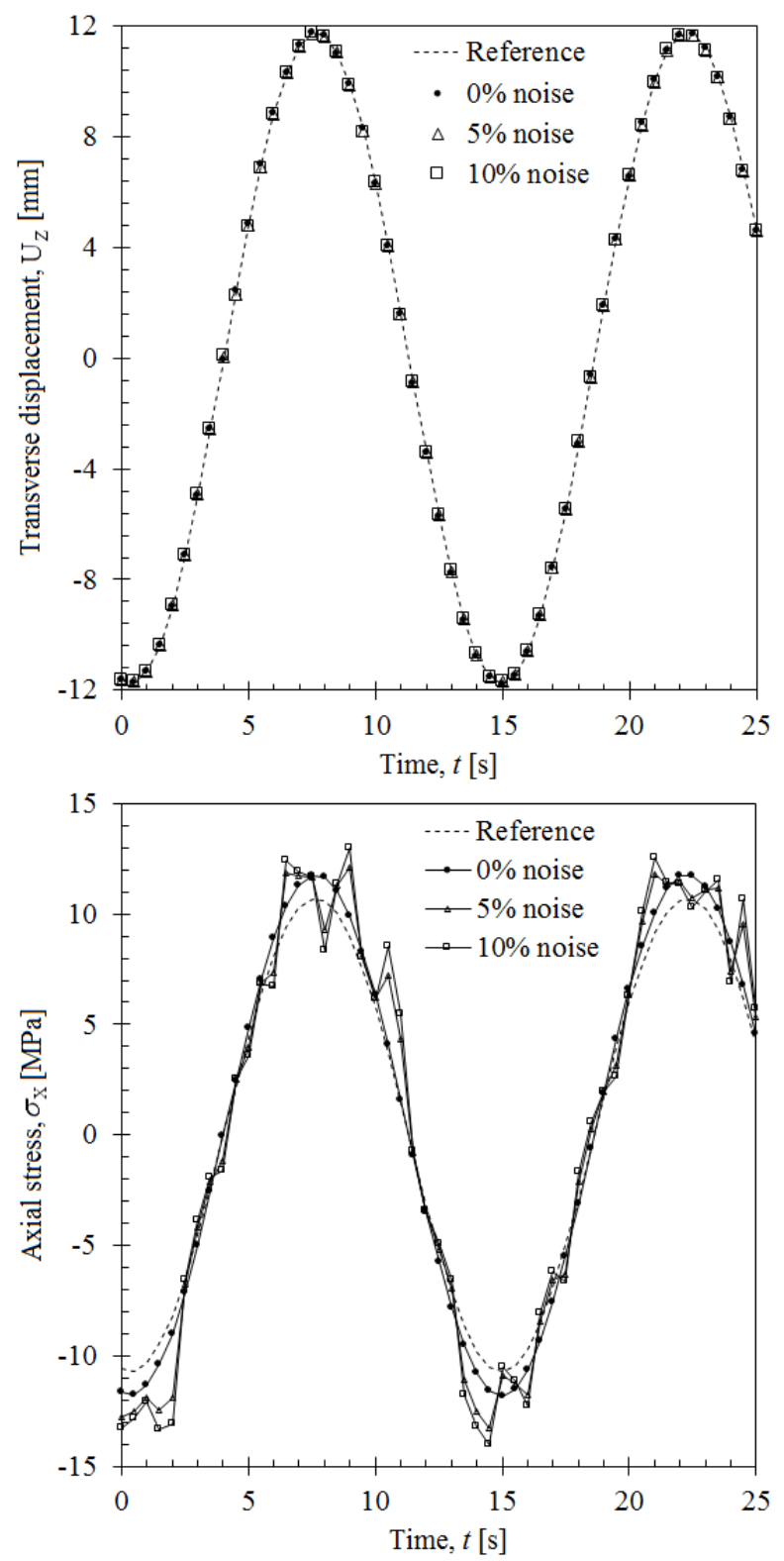

(a)

(b)

Fig. 10. Time history of reference and iFEM $(0 \%, 5 \%$, and $10 \%$ noise $)$ solutions at position $(52.2,0,-19)$ for one-to-one strain data: (a) Transverse displacement, $\mathrm{U}_{\mathrm{Z}}$; (b) Axial stress, $\sigma_{\mathrm{X}}$. 


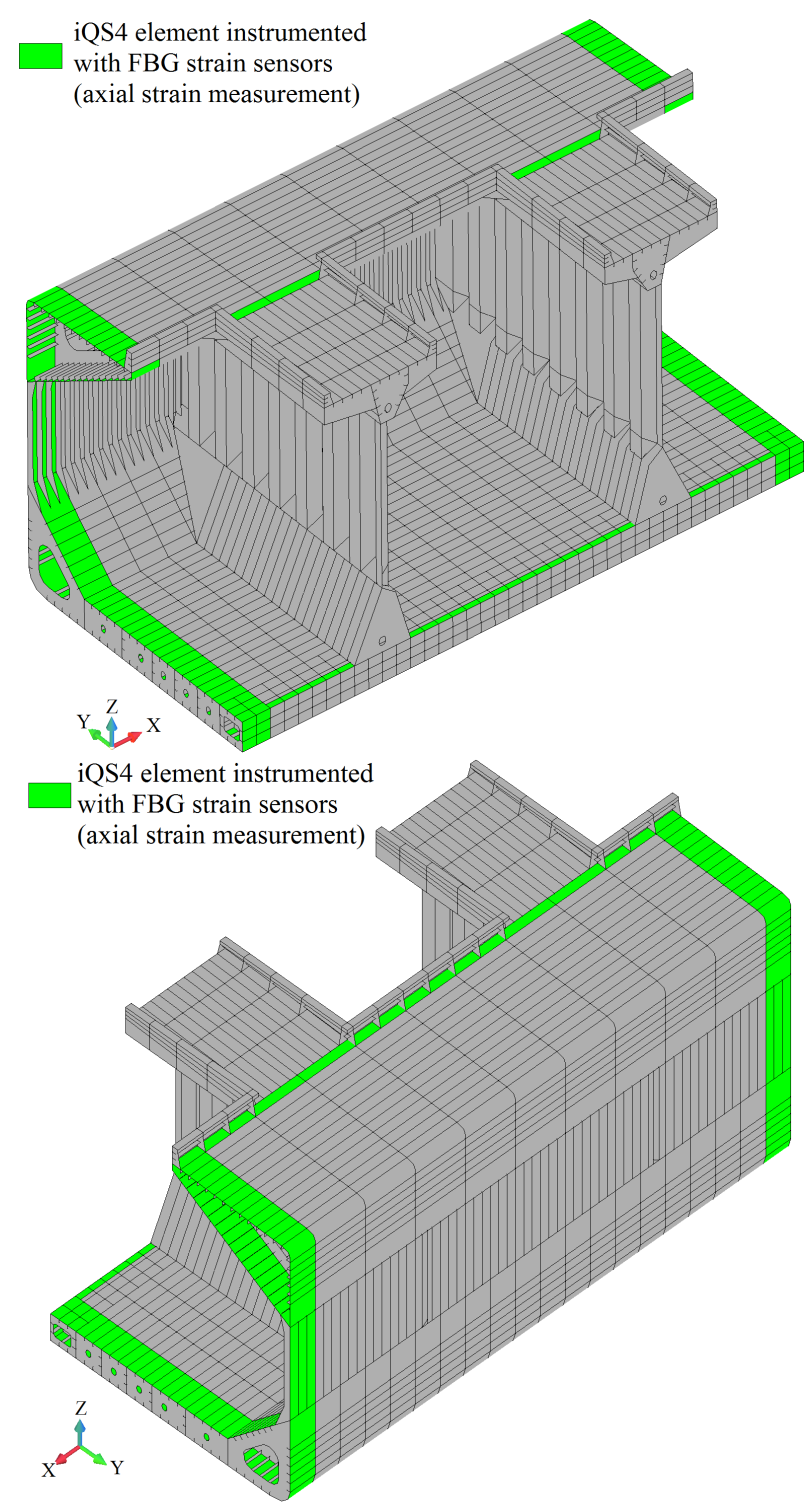

Fig. 11. The iQS4 model of one-fourth of parallel mid-body instrumented with FBG sensor network. 

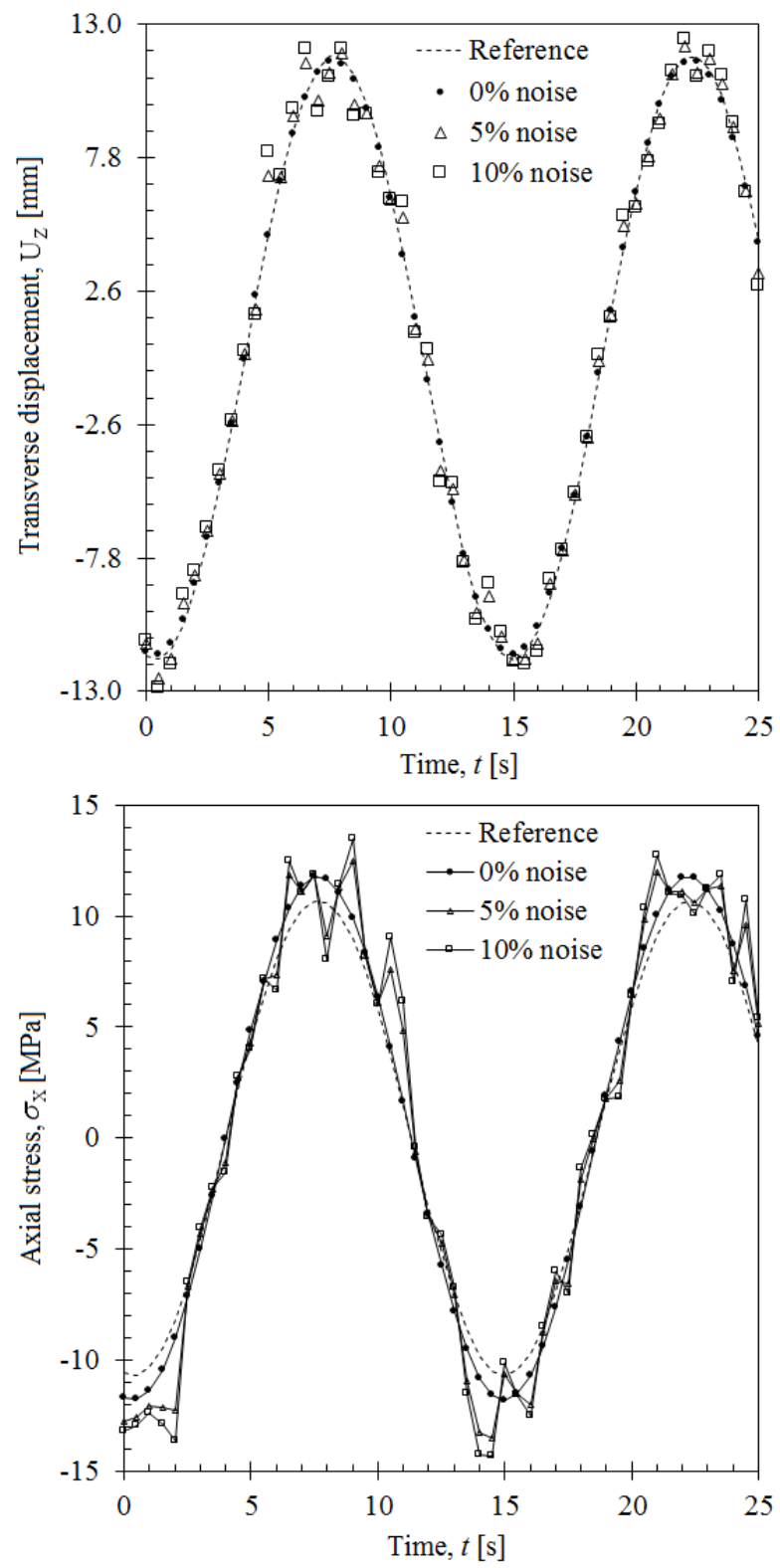

(a)

(b)

Fig. 12. Time history of reference and iFEM $(0 \%, 5 \%$, and $10 \%$ noise $)$ solutions at position $(52.2,0,-19)$ for iQS4 model in Fig. 10: (a) Transverse displacement, $\mathrm{U}_{\mathrm{Z}}$; (b) Axial stress, $\sigma_{\mathrm{X}}$. 

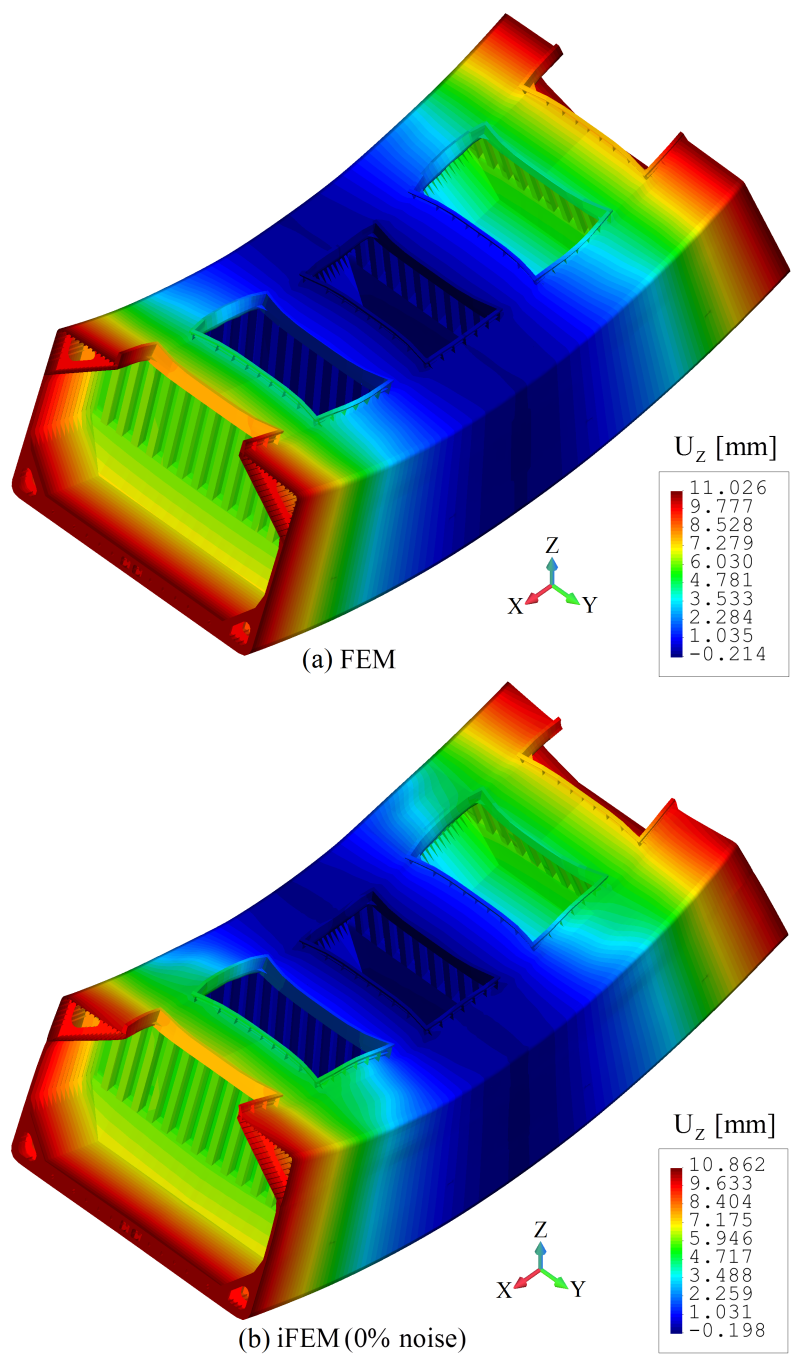


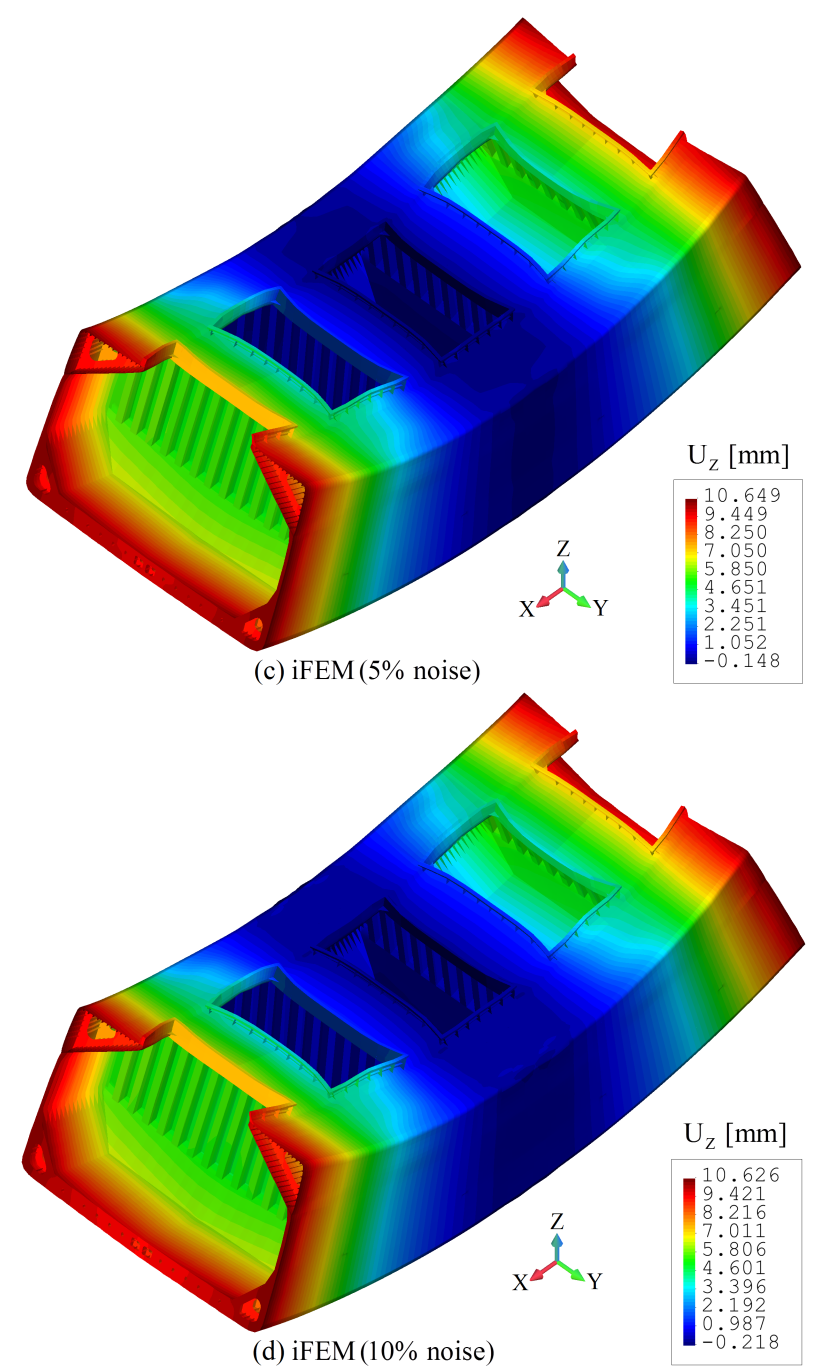

Fig. 13. Contour plots of $U_{z}$ at time $t=8.5 \mathrm{~s}$ for iQS4 model with FBG sensors in Fig. 10: (a) FEM solution; iFEM solution with percent noise in strain measurement: (b) $0 \%$; (c) $5 \%$; (d) $10 \%$. 

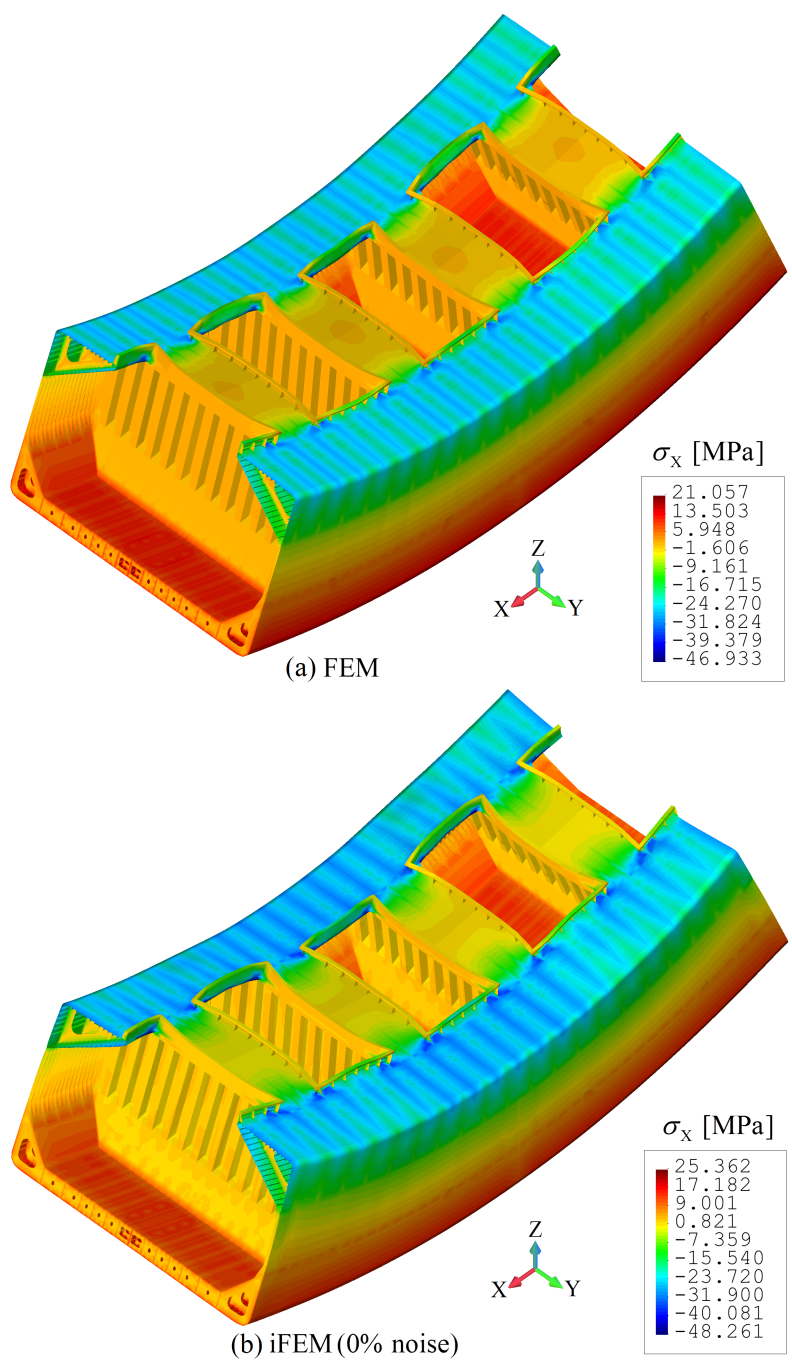


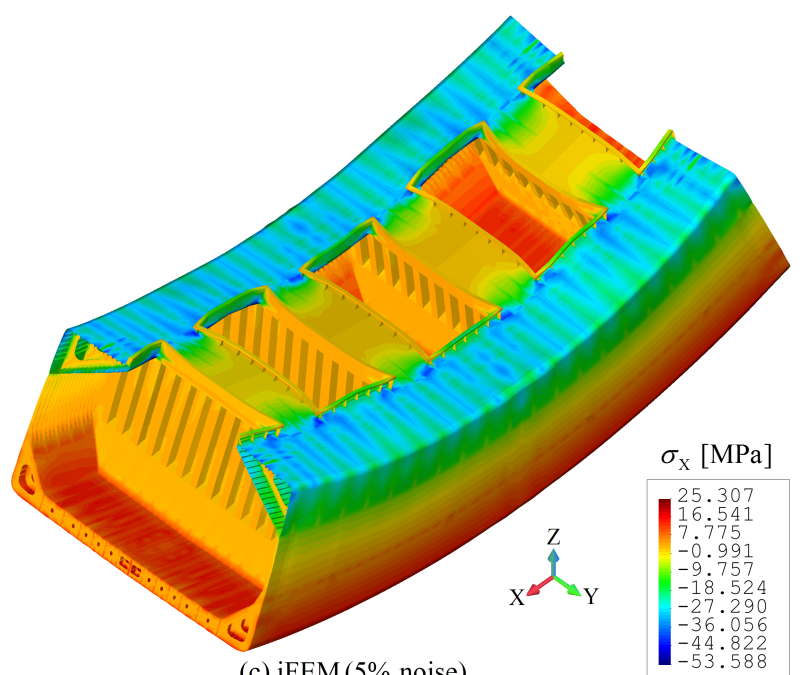

(c) $\operatorname{iFEM}(5 \%$ noise $)$

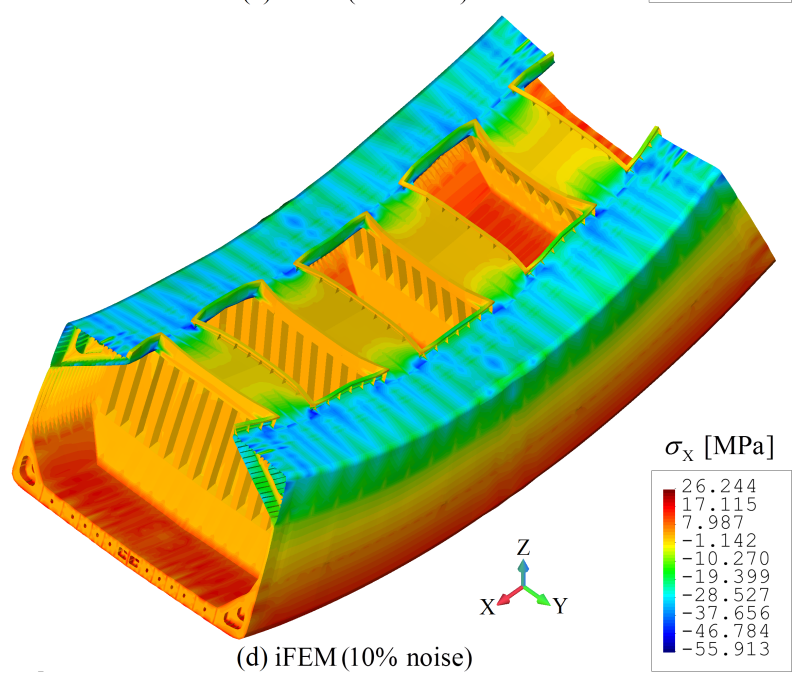

Fig. 14. Contour plots of $\sigma_{\mathrm{X}}$ at time $\mathrm{t}=8.5 \mathrm{~s}$ for iQS4 model with FBG sensors in Fig. 10: (a) FEM solution; iFEM solution with percent noise in strain measurement: (b) $0 \%$; (c) $5 \%$; (d) $10 \%$. 